\title{
Sole use of dexmedetomidine for sedation and analgesia in patients undergoing endovenous thermal ablation for incompetent saphenous veins
}

\author{
Jin Ho Hwang ${ }^{1}$, Il Soo Chang ${ }^{1}$, Sang Woo Park ${ }^{1}$, Won-Kyoung Kwon², Jae Joon Hwang ${ }^{3}$ \\ ${ }^{1}$ Department of Radiology, ${ }^{2}$ Department of Anesthesiology, ${ }^{3}$ Department of Thoracic and Cardiovascular Surgery, Konkuk University School of \\ Medicine, Seoul, Republic of Korea \\ Contributions: (I) Conception and design: JH Hwang, IS Chang, SW Park, WK Kwon; (II) Administrative support: None; (III) Provision of study \\ materials or patients: None; (IV) Collection and assembly of data: JH Hwang, IS Chang, WK Kwon; (V) Data analysis and interpretation: JH \\ Hwang, IS Chang, SW Park, JJ Hwang; (VI) Manuscript writing: All authors; (VII) Final approval of manuscript: All authors. \\ Correspondence to: Jae Joon Hwang, MD, PhD, Professor. Department of Thoracic and Cardiovascular Surgery, Konkuk University School of \\ Medicine, 120-1 Neungdong-ro, Hwayang-dong, Gwangjin-gu, Seoul 143-729, Republic of Korea. Email: hjj@kuh.ac.kr.
}

\begin{abstract}
Background: Dexmedetomidine have both sedative and analgesic properties without respiratorydepressant effect. This study aims to evaluate the safety and effectiveness of sedation and analgesia using dexmedetomidine for the endovenous treatment of varicose veins.

Methods: This study included 88 patients (male $=38$, female $=50$; mean age, 48.7 years) who underwent endovenous laser or radiofrequency ablation of saphenous vein. At the beginning of sedation, dexmedetomidine was administered intravenously to all patients with a loading dose of $1 \mu \mathrm{g} / \mathrm{kg}$ over 15 minutes, which was followed by a maintenance dose of $0.2 \mu \mathrm{g} / \mathrm{kg} / \mathrm{h}$ throughout the procedure. Peripheral oxygen saturation, systolic and diastolic blood pressure, heart rate and respiratory rate, and the induction and recovery time were assessed. The degree of pain was recorded by using a 10-point visual analog scale after the procedure. Adverse events associated with sedation/analgesia were also recorded.

Results: Eighty-five patients responded adequately to sole use of dexmedetomidine. The mean induction time was 17.5 minutes. The mean visual analog scale pain score during the procedure was 2.3 \pm 2.0 . Maximum pain scores of 4 (discomforting) or less were recorded in 69 (78.4\%) patients. Six (6.8\%) patients complained of pain scores in excess of 7 . Systolic and diastolic blood pressure and heart rate drop between 0 and 15 minutes were $18.2 / 9.3 \mathrm{mmHg}$ and 13.5 beat/min, respectively. Three (3.5\%) patients required cessation of infusion due to significant decrease in of blood pressure or heart rate. There was no hypoxic or resuscitation event during the procedures.
\end{abstract}

Conclusions: Dexmedetomidine can provide excellent sedative and analgesic effect during endovenous thermal ablation.

Keywords: Dexmedetomidine; varicose vein; endovenous thermal ablation

Submitted Sep 03, 2019. Accepted for publication Oct 10, 2019.

doi: 10.21037/atm.2019.10.104

View this article at: http://dx.doi.org/10.21037/atm.2019.10.104

\section{Introduction}

Endovenous thermal ablation including laser and radiofrequency ablation (RFA) is a highly effective treatment in patients with incompetent saphenous vein (1). During these procedures, the administration of sedatives is needed to reduce patient anxiety and procedural pain including tumescent anesthesia accompanying multiple needle punctures along the course of the target vein (2). When office-based surgery similar to endovenous thermal ablation is performed, several drugs including midazolam, 
Table 1 Demographic and clinical characteristics

\begin{tabular}{lc}
\hline Characteristic & Value \\
\hline Age (years) & $48.7 \pm 15.0$ (range, 22-77) \\
Sex & 38 \\
Male & 50 \\
Female & $64.8 \pm 10.0$ (range, 46-96) \\
Weight (kg) & 114 (right:left $=57: 57)$ \\
Treated vein & 30 (right:left $=15: 15)$ \\
GSV & 1 \\
SSV & 2 \\
Giacomini & 1 \\
Perforator & 1 \\
AASV &
\end{tabular}

Values presented as mean \pm standard deviation or number of patients. AASV, anterior accessory saphenous vein; GSV, great saphenous vein; SSV, small saphenous vein.

fentanyl, and propofol are most commonly used for sedation and analgesia. However, sometimes sedation-related adverse events such as respiratory depression occur when conscious sedation is carried out by a non-anesthesiologist physician using these drugs, especially in high doses. Therefore, sedatives without respiratory depression are of increasing interest to non-anesthesiologists performing office-based surgery.

Since dexmedetomidine is a highly selective $\alpha_{2}$-adrenergic receptor agonist with both sedative and analgesic properties without respiratory-depressant effect (3), its "off-label" use has been increasing for office-based procedures (4). Although the feasibility of dexmedetomidine use has been shown in many studies, little has been investigated for procedural sedation during endovenous thermal ablation. The purpose of this study was to demonstrate the suitability of dexmedetomidine as the sole sedative agent in the treatment of incompetent saphenous vein using endovenous thermal ablation, to document the hemodynamic and respiratory effects, and to assess the adequacy of the sedative effect during the procedure.

\section{Methods}

After obtaining Institutional Review Board approval (KUH1140121) for observational study and informed consent, a retrospective medical record review was conducted of 88 patients (male $=38$, female $=50$; mean age, 48.7 years; range, $22-77$ years) with duplex ultrasonographyconfirmed great or small saphenous vein (SSV) insufficiency. They underwent endovenous thermal ablation including endovenous laser ablation (EVLA) $(n=7)$ or RFA $(n=81)$ for varicose veins using dexmedetomidine for sedation and analgesia between April 2014 and January 2015. Exclusion criteria included patients under 18 years of age and those with advanced heart block, allergy to dexmedetomidine, heart rate $<50$ beats per minute (bpm), hypotension (systolic blood pressure $<90 \mathrm{mmHg}$ ), hypovolemia, and American Society of Anesthesiologists III or IV. Demographic data of the enrolled patients are illustrated in Table 1 .

After obtaining written informed consent, the patient was brought to the angiographic suite. The procedure room had a tilting table, and the patient was placed in the supine position for the treatment of the great saphenous vein (GSV) or in the prone position for the treatment of SSV. Oxygen ( $3 \mathrm{~L} / \mathrm{min}$ ) was given through an oxygen mask with a reservoir bag.

Intravenous administration of dexmedetomidine diluted with normal saline to a concentration of $4 \mu \mathrm{g} / \mathrm{mL}$ was started. At the start of sedation, dexmedetomidine (Precedex ${ }^{\circledR}$, Hospira Inc., Lake Forest, IL, USA) was administered intravenously to all patients at a loading dose of $1 \mu \mathrm{g} / \mathrm{kg} / \mathrm{h}$ over 15 minutes, which was followed by a maintenance dose of $0.2 \mu \mathrm{g} / \mathrm{kg} / \mathrm{h}$ throughout the procedure using an infusion pump (Syringe Pump TE-331, Terumo, Tokyo, Japan). After 15 minutes, if the patient was not sedated sufficiently, midazolam (Bukwang Pharma, Co., Ltd., Seoul, Korea) bolus was administered repeatedly in increments of $1 \mathrm{mg}$ until conscious sedation. The evaluation of quality of sedation was based on the Modified Observer's Assessment of Alertness/Sedation scale (MOAA/S; Table 2) and quality of analgesia was assessed using a 10 -point visual analog scale (VAS), in which 0 represents no pain at all and 10 represents the worst pain imaginable. The target of sedation was to produce an MOAA/S score of 3 or 4 for each patient throughout the duration of their procedure. The following parameters were monitored throughout the procedure by a trained registered nurse: heart rate, systolic and diastolic blood pressure, respiratory rate, peripheral oxygen saturation, and level of sedation. These parameters were recorded before the drug administration (baseline), at the end of loading dose, and during the procedure (every 5 minutes). The induction time (from the start of dexmedetomidine infusion to patient's sedation) and recovery time (from stoppage of 
Table 2 Observer's assessment of alertness/sedation scale

\begin{tabular}{lc}
\hline Definition & Score \\
\hline $\begin{array}{l}\text { Responds readily to name spoken in normal } \\
\text { tone }\end{array}$ & 5 \\
$\begin{array}{l}\text { Lethargic response to name spoken in } \\
\text { normal tone }\end{array}$ & 4 \\
Responds only after name is called out \\
$\begin{array}{l}\text { loudly and/or repeatedly } \\
\text { Responds only after mild prodding or } \\
\text { shaking } \\
\begin{array}{l}\text { Does not respond to mild prodding or } \\
\text { shaking }\end{array}\end{array}$ \\
\hline
\end{tabular}

Table 3 Modified postanesthesia discharge scoring system for determining home readiness

\begin{tabular}{|c|c|c|}
\hline Category & Description & Score \\
\hline \multirow[t]{3}{*}{ Vital signs } & Within $20 \%$ of preoperative value & 2 \\
\hline & $\begin{array}{l}\text { Within } 20-40 \% \text { of preoperative } \\
\text { value }\end{array}$ & 1 \\
\hline & $>40 \%$ of preoperative value & 0 \\
\hline \multirow[t]{3}{*}{ Ambulation } & $\begin{array}{l}\text { Steady gait, no dizziness at } \\
\text { preoperative level }\end{array}$ & 2 \\
\hline & Requires assistance & 1 \\
\hline & Unable to ambulate & 0 \\
\hline \multirow[t]{3}{*}{ Nausea/vomiting } & $\begin{array}{l}\text { Minimal, treated with oral } \\
\text { medication }\end{array}$ & 2 \\
\hline & $\begin{array}{l}\text { Moderate, treated with parenteral } \\
\text { medication }\end{array}$ & 1 \\
\hline & $\begin{array}{l}\text { Continues after repeated } \\
\text { medication }\end{array}$ & 0 \\
\hline \multirow[t]{2}{*}{ Pain } & Acceptable & 2 \\
\hline & Not acceptable & 1 \\
\hline \multirow[t]{3}{*}{ Surgical bleeding } & $\begin{array}{l}\text { Minimal, no dressing change } \\
\text { required }\end{array}$ & 2 \\
\hline & $\begin{array}{l}\text { Moderate, up to two dressing } \\
\text { changes }\end{array}$ & 1 \\
\hline & $\begin{array}{l}\text { Severe, three or more dressing } \\
\text { changes }\end{array}$ & 0 \\
\hline
\end{tabular}

drug administration to recovery of patient's consciousness) was recorded. When systolic blood pressure drops below $80 \mathrm{mmHg}$, rapid infusion of normal saline was performed to elevate the blood pressure. When the heart rate drops below 40 bpm, glycopyrrolate $\left(\right.$ Mobinul $^{\circledR}$, Myungmoon Pharm. Co., Ltd., Seoul, Korea) was used to correct the bradycardia.

Two practitioners (SW Park, IS Chang) were involved in the endovenous procedure. In patients treated with RFA and EVLA, 7 and 4 Fr introducer sheath was inserted in the GSV or/and SSV under ultrasound guidance, respectively. For the treatment of incompetent saphenous vein, either a 1,470-nm diode laser (Diotec, Busan, Korea) or an RFA catheter (ClosureFast ${ }^{\mathrm{TM}}$, Covidien, Mansfield, MA, USA) was used. The tip of the catheter was located $2 \mathrm{~cm}$ below the saphenofemoral or saphenopopliteal junction. After the tumescent anesthesia was applied using the mixture of normal saline and $2 \%$ lidocaine with a ratio of 49:1 under ultrasound guidance, the GSV or/and SSV was (were) ablated. The RFA was performed according to the manufacture's protocol (20-second treatment cycle with $120^{\circ} \mathrm{C}$ heat) and the EVLA was performed with $1,470 \mathrm{~nm}$ bare-tip fiber in pulse mode and 8-watt power ( $1 \mathrm{~mm} /$ second pullback). Adjuvant ambulatory phlebectomy or/and sclerotherapy was/were performed in the same session. Foam $(0.5-1 \%)$ or liquid sodium tetradecyl sulfate (STS) was used for sclerotherapy.

Home readiness after the procedure was determined by the modified postanesthesia discharge scoring system (MPADSS) scale (Table 3). The duration of procedure and the time from the end of the procedure to the moment of scoring above 9 on MPADSS scale were measured. VAS score during the procedure was recorded just before the patient left the angiographic suite after the procedure.

The collected data were analyzed using PASW Statistics 17 (SPSS, Chicago, IL, USA). Data are expressed as mean \pm standard deviation or number of patients (\%). The differences of measured physiologic parameters associated with the use of dexmedetomidine from the baseline were compared by paired $t$-test, depending on the distribution of the data. A P value $<0.05$ was considered statistically significant.

\section{Results}

A total of 148 endovenous thermal ablations from 88 patients were included in this study. In all patients, endovenous thermal ablation was completed and all patients responded adequately to sole use of dexmedetomidine, except for 3 patients (3.4\%), who showed a resistance to the drug and required supplementation with midazolam for sufficient sedation. The lowest Observer's Assessment 
Table 4 Procedure-related parameters

\begin{tabular}{ll}
\hline Parameters & Value \\
\hline Mean induction time (min) & $17.5 \pm 7.0$ (range, 8-60) \\
Mean sedation time (min) & $56.7 \pm 22.0$ (range, 25-137) \\
Mean procedure time (min) & $43.3 \pm 19.0$ (range, 12-109) \\
Mean recovery time (min) & $35.0 \pm 17.0$ (range, 5-88) \\
\hline
\end{tabular}

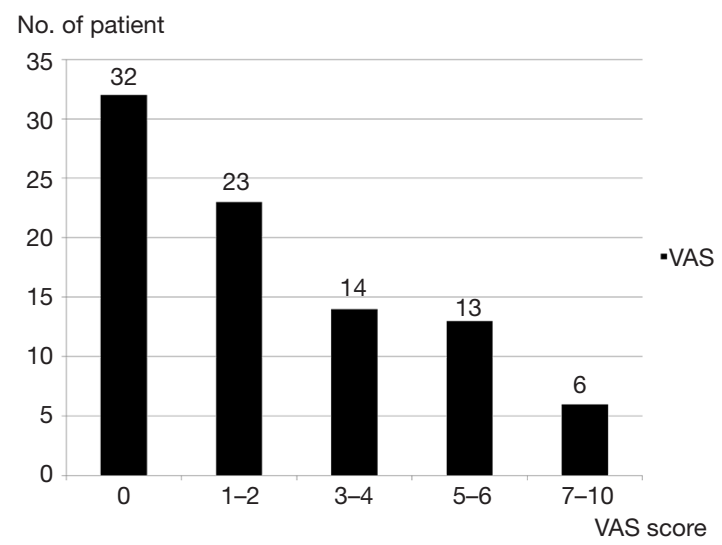

Figure 1 Mean visual analog scale (VAS) scores during the procedure.

of Alertness/Sedation Scale score during the study was 3 points.

Mean induction, sedation, procedure, and recovery time were $17.5 \pm 7.0,56.7 \pm 22.0,43.3 \pm 19.0,35.0 \pm 17.0$ minutes, respectively. These parameters are presented in Table 4.

Mean VAS was $2.3 \pm 2.0$ (range, 0-10). Maximum pain scores of 4 (discomforting) or less were recorded in 69 (78.4\%) patients. Six $(6.8 \%)$ patients complained of pain scores in excess of 7 (horrible) (Figure 1).

The variation of the hemodynamic parameters including heart rate, systolic and diastolic blood pressure, or physiologic parameters such as respiratory rate and oxygen saturation are presented in Table 5. A statistically significant decrease in heart rate, systolic and diastolic blood pressure occurred upon completion of the loading dose compared to baseline $(\mathrm{P}<0.001)$ (Figures 2,3). However, there were no significant interval changes in heart rate and blood pressure during the maintenance dose period. Mean values of decrease in heart rate, systolic and diastolic blood pressure were $13.5 \pm 10.0 \mathrm{bpm}$ and $18.2 \pm 20.0$ and $9.3 \pm 13.0 \mathrm{mmHg}$, respectively. Respiratory rate was stable and none of the patients experienced a hypoxic event during the whole procedure (Figures 4,5).

Glycopyrrolate was administered in four patients $(4.7 \%)$ in order to correct bradycardia. Three of these patients recovered normal sinus rhythm, but cessation of dexmedetomidine was required in one patient due to persistent bradycardia even after glycopyrrolate administration.

Three patients $(3.5 \%)$ required cessation of infusion due to tachycardia $(n=1)$, bradycardia $(<40 \mathrm{bpm})(\mathrm{n}=1)$, or irritability $(\mathrm{n}=1)$. Heart rate rapidly increased to $158 \mathrm{bpm}$ within 5 minutes after administration of dexmedetomidine in one patient who showed tachycardia. The heart rate returned to $82 \mathrm{bpm}$ within 10 minutes after discontinuation of drug infusion without any other therapeutic measures.

One patient presented persistent bradycardia (37 bpm) that was not corrected with glycopyrrolate in 20 minutes after sedation and required immediate discontinuation of dexmedetomidine infusion. The last patient showed severe paradoxical agitation during the loading dose period, and administration of dexmedetomidine had to be discontinued.

Nine patients $(10.6 \%)$ had minor complications including nausea $(\mathrm{n}=5,5.9 \%)$, dizziness $(\mathrm{n}=3,3.5 \%)$, and headache $(n=1,1.2 \%)$ after the procedure, all of whom had resolution of symptoms without additional treatment.

\section{Discussion}

Because more and more vascular procedures are currently performed on an outpatient basis with local or regional anesthesia, effective and safe anesthetic agents and techniques are frequently needed for maximal patient comfort. Non-anesthesiologists including surgeons, endoscopists, and interventional radiologists have been interested in reducing the procedural pain and anxiety along with safety. The combination of midazolam and fentanyl is known to provide sufficient sedative and analgesic effect during various interventional procedures associated with discomfort (5). However, these drugs may be associated with an increased risk of respiratory depression and oxygen desaturation. Since dexmedetomidine produces sedation, moderate analgesia, and anxiolysis, and causes minimal respiratory depression, it can be considered as a preferred sedative for use by the non-anesthesiologists. Although the US Food and Drug Administration approved dexmedetomidine in 1999 for sedation in intubated patients in intensive care not to exceed 24 hours (6), off-label use of this drug is becoming more common in various fields of medicine due to its excellent sedative ability and safety 
Table 5 Hemodynamic and respiratory parameters

\begin{tabular}{|c|c|c|c|c|c|c|c|c|}
\hline Variable & Baseline & $15 \min (E L D)$ & $20 \min$ & $25 \min$ & $30 \mathrm{~min}$ & Stop & \multicolumn{2}{|c|}{$P$ value } \\
\hline SBP & $133.8 \pm 19.1$ & $120.5 \pm 21.6$ & $119.5 \pm 21.9$ & $116.3 \pm 22.7$ & $117.2 \pm 21.2$ & $118.0 \pm 20.8$ & $<0.001$ & $<0.001$ \\
\hline $\mathrm{SpO}_{2}$ & $98.5 \pm 2.0$ & $99.4 \pm 0.8$ & $99.4 \pm 0.7$ & $99.3 \pm 0.7$ & $99.0 \pm 1.3$ & $98.9 \pm 1.3$ & - & - \\
\hline
\end{tabular}

ELD, end of loading dose; DBP, diastolic blood pressure; RR, respiratory rate; SBP, systolic blood pressure; $\mathrm{SpO}_{2}$, oxygen saturation.

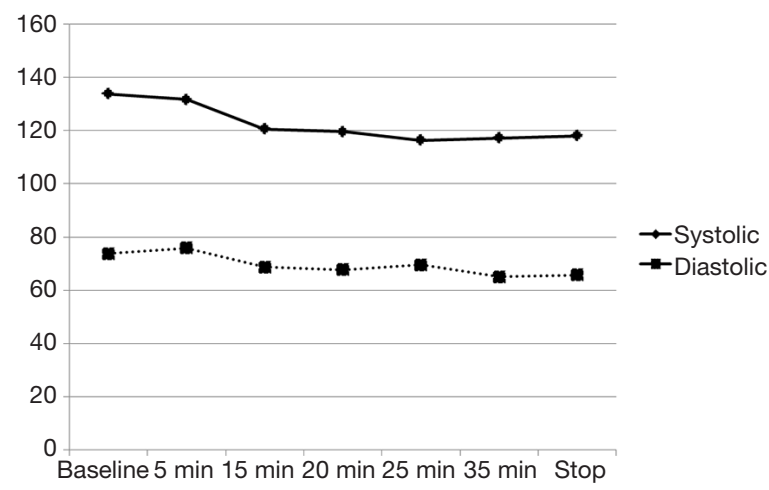

Figure 2 The changes in mean systolic and diastolic blood pressure during the procedure.

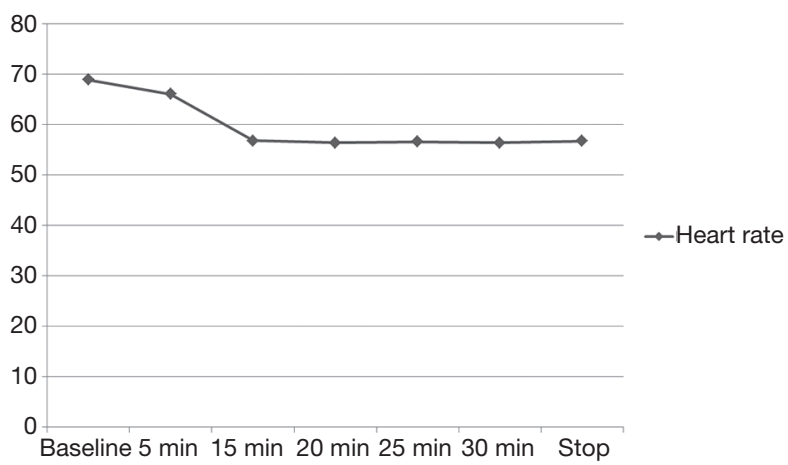

Figure 3 The changes in mean heart rate during the procedure.

$(4,7)$. Dexmedetomidine has been used for sedation during laryngeal surgery (8), awake fiberoptic intubation (9), flexible bronchoscopy (10), percutaneous carotid artery stenting (11), and upper gastrointestinal endoscopy (12). Most of these studies showed that dexmedetomidine provided sufficient sedation and analgesia during
$\mathrm{RR}$

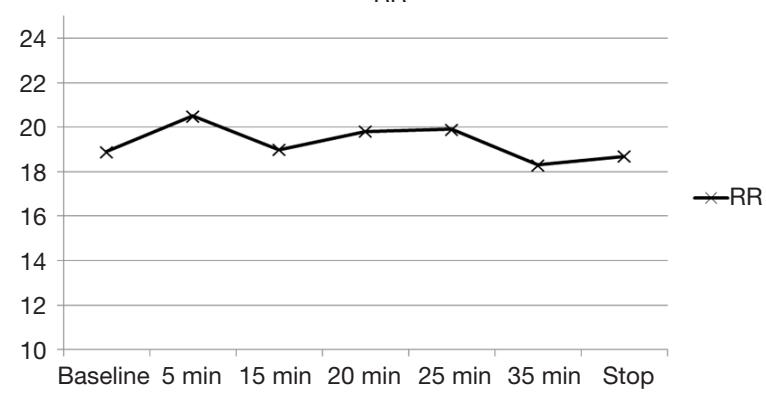

Figure 4 The changes in mean respiratory rate. RR, respiratory rate.

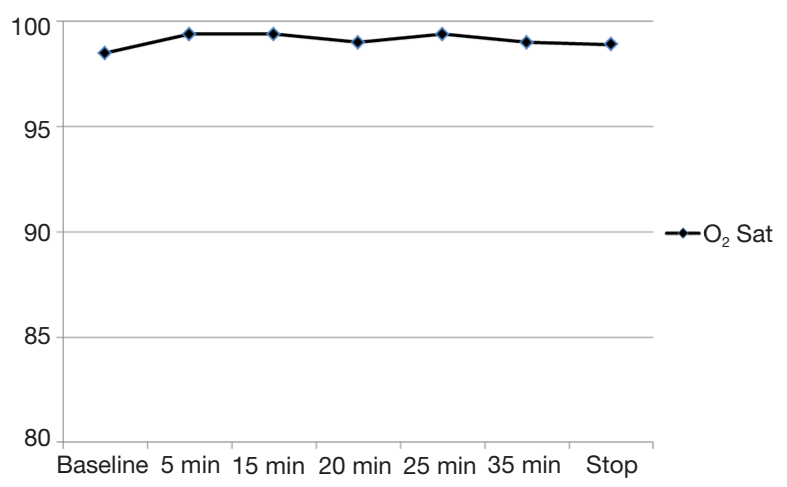

Figure 5 The changes in mean $\mathrm{O}_{2}$ saturation during the procedure.

the procedure. The results of the present study also demonstrated that the sole use of dexmedetomidine can be a safe and an effective anesthetic method for patients undergoing endovenous thermal ablation. Endovenous thermal ablation is almost always accompanied by repeated painful skin punctures with needle and perivascular injections for tumescent anesthesia that desensitize patients from skin burn and severe heating pain, and protect patients 
from nerve injury. Once the patients are sedated and relaxed after administration of dexmedetomidine, they can tolerate such noxious stimuli during the procedure. Therefore, dexmedetomidine can be a useful anesthetic adjunct for sedation during infiltration of local anesthesia in the desired area (4).

Dexmedetomidine affords a unique form of sedation that is characterized by easy arousability and cooperative sedation which allows communication with the patient at stimulation, even though the patient may appear to be asleep (13). These characteristics are important during endovenous thermal ablation, because a patient who has venous insufficiency in both great and SSVs will be required to change position during the procedure. Usually GSV is ablated in supine position and SSV in prone position. When GSV and SSV are to be ablated in the same treatment session, the patient's position has to be changed in the middle of the procedure. Therefore, cooperative sedation can be another benefit of dexmedetomidine during endovenous thermal ablation.

The heart rate and blood pressure demonstrated a statistically significant decrease after the loading dose of dexmedetomidine in most patients in the current study. Bradycardia and hypotension are expected effects, resulting from sympatholytic activity (14). Popat et al. described that most events of bradycardia and hypotension occurred shortly after administration of the loading dose (4). Most of the patients in the present study, however, showed hemodynamic stability during the procedure and only four patients $(4.5 \%)$ had a significant drop in heart rate (<40 bpm), who required glycopyrrolate to treat bradycardia. Among these, three patients (3.4\%) recovered normal sinus rhythm after treatment with glycopyrrolate, but one patient underwent cessation of dexmedetomidine infusion due to uncontrolled bradycardia. Because of these properties, it is recommended that dexmedetomidine should be used with caution in patients with advanced heart block or severe ventricular dysfunction or hypovolemia (15). In the present study, other minor adverse events of sedation using dexmedetomidine included nausea, dizziness, headache, paradoxical agitation, and tachycardia, all of which resolved spontaneously without additional therapeutic measures.

Peripheral hemoglobin oxygen saturation was not affected by dexmedetomidine infusion. This finding is consistent with the study by Ebert and coworkers (16).

The desired level of sedation was achieved in most of the patients (96.5\%), but three patients needed supplementary midazolam to achieve an adequate level of sedation. It is known that about $10-20 \%$ of patients sedated with dexmedetomidine require supplementation with an additional sedative agent (17).

Compared with frequently used sedatives such as midazolam and propofol, dexmedetomidine has a relatively long recovery time. In some studies, time required to reach home readiness was $85 \pm 74$ minutes (18) and $82.2 \pm 24.3$ minutes (19). In the present study, there was concern about this distinct property and considered the importance of early ambulation to prevent deep vein thrombosis after the treatment of varicose veins. Therefore, dexmedetomidine infusion was discontinued about 20 minutes before the end of the procedure to shorten the recovery time. Consequently, the recovery time of patients in the current study ( $35 \pm 17$ minutes) could be shorter than that reported in previous studies.

\section{Conclusions}

In conclusion, dexmedetomidine provides some welldocumented properties including sedation, analgesia, anxiolysis, and preservation of respiratory function. Therefore, this sedative can also be suitable for endovenous thermal ablation of incompetent saphenous veins when coupled with local anesthesia. This report may support a promising result in a kind of "off-label" clinical scenario. Dexmedetomidine, utilized as a single intravenous agent, virtually produced no undesirable respiratory effects, while allowing for adequate sedation in the majority of patients. Although this study supports a good clinical profile, it is a limitation that the present study did not directly compare dexmedetomidine with other sedatives such as propofol or midazolam. Therefore, further comparison studies will be required to validate the use of dexmedetomidine during endovenous procedure.

\section{Acknowledgments}

None.

\section{Footnote}

Conflicts of Interest: The authors have no conflicts of interest to declare

Ethical Statement: The authors are accountable for all aspects of the work in ensuring that questions related to the accuracy or integrity of any part of the work are 
appropriately investigated and resolved. This study was approved by the Institutional Review Board (No. KUH1140121) and informed consent was obtained from all patients involved.

\section{References}

1. van den Bos R, Arends L, Kockaert M, et al. Endovenous therapies of lower extremity varicosities: a meta-analysis. J Vasc Surg 2009;49:230-9.

2. Morrison N, Gibson K, McEnroe S, et al. Randomized trial comparing cyanoacrylate embolization and radiofrequency ablation for incompetent great saphenous veins (VeClose). J Vasc Surg 2015;61:985-94.

3. Paris A, Tonner PH. Dexmedetomidine in anaesthesia. Curr Opin Anaesthesiol 2005;18:412-8.

4. Popat KU, Purugganan RV, Malik I. Off-Label Uses of Dexmedetomidine. Advances in Anesthesia 2006;24:177-92.

5. Skehan SJ, Malone DE, Buckley N, et al. Sedation and analgesia in adult patients: evaluation of a stageddose system based on body weight for use in abdominal interventional radiology. Radiology 2000;216:653-9.

6. Jacobi J, Fraser GL, Coursin DB, et al. Clinical practice guidelines for the sustained use of sedatives and analgesics in the critically ill adult. Crit Care Med 2002;30:119-41. Erratum in: Crit Care Med 2002;30:726.

7. Venn RM, Karol MD, Grounds RM. Pharmacokinetics of dexmedetomidine infusions for sedation of postoperative patients requiring intensive caret. $\mathrm{Br} \mathrm{J}$ Anaesth 2002;88:669-75.

8. Jense RJ, Souter K, Davies J, et al. Dexmedetomidine sedation for laryngeal framework surgery. Ann Otol Rhinol Laryngol 2008;117:659-64.

9. Madhere M, Vangura D, Saidov A. Dexmedetomidine as sole agent for awake fiberoptic intubation in a patient with local anesthetic allergy. J Anesth 2011;25:592-4.

Cite this article as: Hwang JH, Chang IS, Park SW, Kwon WK, Hwang JJ. Sole use of dexmedetomidine for sedation and analgesia in patients undergoing endovenous thermal ablation for incompetent saphenous veins. Ann Transl Med 2019;7(22):638. doi: $10.21037 /$ atm.2019.10.104
10. Ni YL, Lo YL, Lin TY, et al. Conscious sedation reduces patient discomfort and improves satisfaction in flexible bronchoscopy. Chang Gung Med J 2010;33:443-52.

11. Cata JP, Folch E. Dexmedetomidine as sole sedative during percutaneous carotid artery stenting in a patient with severe chronic obstructive pulmonary disease. Minerva Anestesiol 2009;75:668-71.

12. Wu W, Chen Q, Zhang LC, et al. Dexmedetomidine versus midazolam for sedation in upper gastrointestinal endoscopy. J Int Med Res 2014;42:516-22.

13. Abdelmalak B, Makary L, Hoban J, et al. Dexmedetomidine as sole sedative for awake intubation in management of the critical airway. J Clin Anesth 2007;19:370-3.

14. Scheinin B, Lindgren L, Randell T, et al. Dexmedetomidine attenuates sympathoadrenal responses to tracheal intubation and reduces the need for thiopentone and peroperative fentanyl. Br J Anaesth 1992;68:126-31.

15. Chrysostomou C, Schmitt CG. Dexmedetomidine: sedation, analgesia and beyond. Expert Opin Drug Metab Toxicol 2008;4:619-27.

16. Ebert TJ, Hall JE, Barney JA, et al. The effects of increasing plasma concentrations of dexmedetomidine in humans. Anesthesiology 2000;93:382-94.

17. Riker RR, Fraser GL. Adverse events associated with sedatives, analgesics, and other drugs that provide patient comfort in the intensive care unit. Pharmacotherapy 2005;25:8S-18S.

18. Jalowiecki P, Rudner R, Gonciarz M, et al. Sole use of dexmedetomidine has limited utility for conscious sedation during outpatient colonoscopy. Anesthesiology 2005;103:269-73.

19. Makary L, Vornik V, Finn R, et al. Prolonged recovery associated with dexmedetomidine when used as a sole sedative agent in office-based oral and maxillofacial surgery procedures. J Oral Maxillofac Surg 2010;68:386-91. 